\title{
Time to Start Up: CT-Basted Radiomics in Children's Lung Diseases
}

\author{
Zhang $\mathbf{Y}^{\mathbf{1}}$, Ma $\mathbf{X}^{\mathbf{1}}$ and Zhao $\mathbf{C}^{2 *}$ \\ 'Department of Respiratory Medicine, Qilu Children's \\ Hospital of Shandong University, China \\ ${ }^{2}$ Department of Pediatrics, Qilu Hospital of Shandong \\ University, China \\ *Corresponding author: Cuifen Zhao, Department of \\ Pediatrics, Qilu Hospital of Shandong University, J inan \\ 250012, China
}

Received: April 19, 2021; Accepted: May 08, 2021;

Published: May 15, 2021

\section{Editorial}

Radiomics is a new interdisciplinary field and a fusion product consisting by large data technology and medical image to aid diagnosis. Radiomics can gather information from different medical imaging (i.e. CT, PET, MRI, ultrasound) for deeper excavation, predict and analyse to quantify disease characteristics, establish disease models, and identify new diagnostic and prognostic biomarkers to assist physicians in making the most accurate diagnosis [1,2]. Recently, it has evolved into a method which consisted of imaging, gene, and clinical information for auxiliary diagnosis, analysis and prediction. CT-basted radiomics has significant advantages in the study of lung diseases due to its high resolution for lung tissue, which represents a great potential for accurate diagnosis and treatment of lung diseases $[3,4]$. Accurate diagnosis is a difficult problem in pediatric imaging. In the era of precision medicine, the development of CT-basted radiomics brings challenges and opportunities for the accurate diagnosis and treatment of children's lung diseases.

At present, the application of radiomics to the thorax is almost exclusively focused on lung cancer, specifically, the detection of lung cancer, prediction of histology and subtype, prediction of prognosis, and assessment of treatment effect [5], which has shown independent prognosis and prediction capacity in many tumors and played a very important role in increasing the accuracy of diagnosis, reducing the application rate of invasive examination, and assessing the risk of lung cancer progression [6,7]. In addition, imageology has also been used to predict lung cancer gene phenotype and mutation [8].

Some studies have been carried out in pulmonary inflammation disease including infectious pneumonia, interstitial pneumonie, chronic lung injury and chronic pulmonary diseases. By extracting characteristics of image area of lesion and using computer-assisted texture-based image analysis, quantitative assessment of highresolution computed tomography and disease assessment can be realized adequately in general interstitial pneumonie [9]. the accuracy rate of classification for the image area of ground-glass opacity lesion was $70.7 \%$.In addition, different image group labels can extract different image group characteristics, which can be used to identify chronic lung injury and pneumonia [10].
Chronic Obstructive Pulmonary Disease (COPD), as a common disease in respiratory department, is caused by small airway disease (obstructive bronchitis) and lung parenchymal destruction (emphysema). However, the proportions of lesion in COPD patients were different [11]. As the development of CT-basted radiomics, the mathematical model of airway function based on standard vital capacity can be used to analyze the existence and severity of emphysema in patients with COPD [12]. The volume of pulmonary emphysema and air-trapping retention in patients with COPD can also be quantified and positioned relatively by using the parameters of the low attenuation area in CT scans [13]. The visual manifestations and severity of emphysema, which may reflect the severity of small airway disease, are significantly correlated with the risk of death $[14,15]$. The study of Charbonnier JP et al. also has been proved that the parametric spectrum of lung (PRM) is a tool for the classification of quantitative density of COPD [16]. Cho MH et al. showed the relationship between gene and image subtypes in 12031 patients with COPD, which opened a new field for the differential genetics of COPD phenotype [17].

Asthma, as a heterogeneous disease, is easily confused with COPD and can benefit from the classification of subtypes. Improving the prognosis of asthmatic patients by using personalized clinical and imaging biomarkers has been one of the primary goals of the Severe Asthma Research Program (SARP) Project [18]. Quantitative study of CT providing structural and functional information of lung has been a useful tool for the study of asthma $[19,20]$, This technique can identify the unique structure and functional phenotype of asthma and COPD successfully [21]. Research findings airway remodeling and air retention in Quantitative study of CT were associated with lung function, severity of asthma and histology, which can be used to distinguish asthma subgroups and served as the basis for the development of new therapies [22,23].

Quantitative analysis of chest CT images can identify and quantify Interstitial Lung Disease (ILD) [24]. The main imaging features of ILD on CT images are ground glass shadow, honeycomb shadow, reticular shadow, and consolidation shadow etc. Pulmonary Quantitative Analysis (QA) of CT images can objectively quantify specific patterns of ILD changes during treatment in patients with SSc-ILD [25]. With the understanding of different modes of IFP, which can find subtle changes, it may be helpful not only to reduce the invasive operation, but also to precisely treat patients and evaluate the therapeutic effects [26].

Therefore, based on the theory and clinical applications of radiomics, CT-basted radiomics will serve as a new radiological analysis tool for treatment prediction in lung diseases of children [27]. However, the application of accurate radiomic in children's lung diseases is rare. Only a few studies have focus on the semiquantitative CT measurements to quantitatively assess the extent of 
air trapping $[28,29]$. The characteristic CT findings in $\mathrm{BO}$ included mosaic air retention, bronchiectasis and atelectasis, which were not evenly distributed throughout the lung [30]. Quantitative detection of $\mathrm{CT}$ in post-transplant $\mathrm{BO}$ patients with air retention is associated with airway obstruction in PFT [28]. A previous study showed the value of the quantitative $\mathrm{CT}$ analysis in predicting severity and longitudinal changes of inhalation lung injury. The quantitative CT analysis could also help to assess pulmonary function by some CT indicators, including Normally Aerated Volume Ratio (NAVR) and Reductively Aerated Volume Ratio (RAVR) [31]. Some studies also focused on unique structural abnormalities in chest CT scans of Bronchopulmonary Dysplasia (BPD) patients, and found that the scope of lesions in image correlates with the clinical manifestations and lung function in children with BPD.

Currently, radiomics has become a challenge in pediatrics and also proposes some problems need to be further solved. Firstly, It is necessary to establish standardization programme for many factors, such as scanning scheme, research method, parameters, and establish multiple diseases and large sample data. Secondly, the stability of image and the accuracy of radiomics model building also needs further study. Lastly, the relationship between radiomics, histopathology, and gene, needs to be further explored in pediatrics.

In summary, radiomics can help to identify new biomarkers, provide new insights for understanding the phenotypic of unknown diseases, reduce or avoid traumatic operations, and provide broad prospects for accurate diagnosis and personalized treatment. Based on the advantages of $\mathrm{CT}$ in lung diseases, we aim to focuse attention on the CT-basted radiomics in lung diseases of children to provide accurate information support for accurate medical of children's lung diseases.

\section{Funding}

This work was supported by Jinan Science Technology Bureau (202019172).

\section{References}

1. Aerts HJ, Velazquez ER, Leijenaar RT, et al. Decoding tumour phenotype by noninvasive imaging using a quantitative radiomics approach. Nat Commun. 2014, 5: 4006 .

2. Su HF, Zhou GF, Xie CM, et al. The rise and development of radiomics. Chinese Medical Journal. 2015; 95: 553-556.

3. Ganeshan B, Panayiotou E, Burnand K, Dizdarevic S, Miles K. Tumour heterogeneity in non-small cell lung carcinoma assessed by CT texture analysis: a potential marker of survival. Eur Radiol. 2012; 22: 796-802.

4. El Naqa I, Grigsby PW, Apte A, et al. Exploring feature-based approaches in PET images for predicting cancer treatment outcomes. Pattern Recognit. 2009; 42: 1162-1171.

5. Hassani C, Varghese BA, Nieva J, Duddalwar V. Radiomics in Pulmonary Lesion Imaging. AJR Am J Roentgenol. 2019; 212: 497-504.

6. Hawkins $\mathrm{S}$, Wang $\mathrm{H}$, Liu $\mathrm{Y}$, et al. Predicting malignant nodules from screening CT scans. J Thorac Oncol. 2016; 11: 2120-2128.

7. Vallieres $M$, Freeman $C R$, Skamene $S R$, et al. A radiomics model from joint FDG-PET and MRI texture features for the prediction of lung metastases in soft-tissue sarcomas of the extremities. Phys Med Biol. 2015; 60: 5471-5496.

8. Liang $\mathrm{T}$, Xie S. A review of Omics Imaging and its application in pulmonary diseases. Journal of China Japan Friendship Hospita. 2019; 33: 35-37.

9. Delorme S, Keller-Reichenbecher M-A, Zuna I, et al. Usual interstitial pneumonia: quantitative assessment of high-resolution computed tomography findings by computer-assisted texture-based image analysis. Investigative radiology. 1997; 32: 566-574

10. Wang W, Song ZJ, Ding H, et al. Imaging studey about distribution sites of lesion areas of pulonary fibrosis induced by paraquat. Chinese Journal of Emergency Medicine. 2011; 6: 614-618.

11. Expert Group of Guideline for Primary Care of Respiratory System Disease. Guidline for primary care of chronic obstructive pulmonary disease (2018). Chinese Journal of General Practitioners. 2018; 17: 856-870.

12. Occhipinti M, Paoletti M, Bartholmai BJ, et al. Spirometric assessment of emphysema presence and severity as measured by quantitative CT and CTbased radiomics in COPD. Respir Res. 2019; 20: 101.

13. Schroeder JD, McKenzie AS, Zach JA, et al. Relationships between airflow obstruction and quantitative CT measurements of emphysema, air trapping, and airways in subjects with and without chronic obstructive pulmonary disease. AJR Am J Roentgenol. 2013; 201: W46-W470.

14. Lynch DA1, Moore CM1, Wilson C et al. CT-based Visual Classification of Emphysema: Association with Mortality in the COPDGene Study. Radiology. 2018; 288: 859-866.

15. Mair G, Maclay J, Miller JJ, et al. Airway dimensions in COPD: relationships with clinical variables. Respir Med. 2010; 104: 1683-1690.

16. Charbonnier JP, Pompe E, Moore $\mathrm{C}$, et al. Airway wall thickening on CT: Relation to smoking status and severity of COPD. Respir Med. 2019; 146: 36-41.

17. Cho MH, Castaldi PJ, Hersh CP, Hobbs BD, Barr RG, Tal-Singer R, et al. A Genome-Wide Association Study of Emphysema and Airway Quantitative Imaging Phenotypes. Am J Respir Crit Care Med. 2015; 192: 559-569.

18. Jarjour NN, Erzurum SC, Bleecker ER, et al. Severe asthma: lessons learned from the National Heart, Lung, and Blood Institute Severe Asthma Research Program. Am J Respir Crit Care Med. 2012; 185: 356-362.

19. DeBoer EM, Spielberg DR, Brody AS. Clinical potential for imaging in patients with asthma and other lung disorders. J Allergy Clin Immunol. 2017; 139: 21-28.

20. Sanghun Choi, Babak Haghighi, Jiwoong Choi, et al. Differentiation of quantitative CT imaging phenotypes in asthma versus COPD. BMJ Open Respir Res. 2017; 4: e000252.

21. Aysola RS, Hoffman EA, Gierada D, et al. Airway remodeling measured by multidetector CT is increased in severe asthma and correlates with pathology. Chest. 2008; 134: 1183-1191.

22. Choi S1, Hoffman EA2, Wenzel SE3, et al. Quantitative computed tomographic imaging-based clustering differentiates asthmatic subgroups with distinctive clinical phenotypes.J Allergy Clin Immunol. 2017; 140: 690-700.

23. Shim SS, Schiebler ML, Evans MD, et al. Lumen area change (Delta Lumen) between inspiratory and expiratory multidetector computed tomography as a measure of severe outcomes in asthmatic patients.J Allergy Clin Immunol. 2018; 142: 1773-1780.

24. Tashkin DP, Volkmann ER, Tseng $\mathrm{CH}$, Kim HJ, Goldin J, Clements $\mathrm{P}$, et al. Relationship between quantitative radiographic assessments of interstitial lung disease and physiological and clinical features of systemic sclerosis. Ann Rheum Dis. 2016; 75: 374-381.

25. Occhipinti M, Bosello S, Sisti LG, et al. Quantitative and semi-quantitative computed tomography analysis of interstitial lung disease associated with systemic sclerosis: A longitudinal evaluation of pulmonary parenchyma and vessels. PLoS One. 2019; 14: e0213444.

26. Wu X, Kim GH, Salisbury ML, et al. Computed Tomographic Biomarkers in Idiopathic Pulmonary Fibrosis. The Future of Quantitative Analysis. Am J Respir Crit Care Med. 2019; 199: 12-21.

27. Van Mastrigt E, Logie $K$, Ciet $P$, et al. Lung CT imaging in patients with bronchopulmonary dysplasia: A systematic review (Review). Pediatr Pulmonol. 2016; 51: 975-986.

28. Kim HG, Shin HJ, Kim YH, et al. Quantitative computed tomography 
assessment of graft-versus-host disease-related bronchiolitis obliterans in children: A pilot feasibility study. Eur Radiol. 2015; 25: 2931-2936.

29. Kim J, Conceptualization, Data curation, et al. Quantitative CT and pulmonary function in children with post-infectious bronchiolitis obliterans. PLoS One. 2019; 14: e0214647.

30. Fischer GB, Sarria EE, Mattiello R, Mocelin HT, Castro-Rodriguez JA. Post infectious bronchiolitis obliterans in children. Paediatr Respir Rev. 2010; 11 233-239.

31. Yaqiong $M$, Shikui Z, Lianping Z, et al. Inhalation lung injury induced by smoke bombs in children:CT manifestations, dynamic evolution features and quantitative analysis. J Thorac Dis. 2018; 10: 5860-5869. 